\title{
Prenatal protection through TLRs
}

The decreased pre- and postnatal exposure to microbial products in industrialized countries has been proposed to contribute to the increased incidence of asthma and allergies, a theory termed the hygiene hypothesis. For example, epidemiology studies suggest that childhood exposure to farming environments protects against the development of allergic diseases, and the Gram-negative

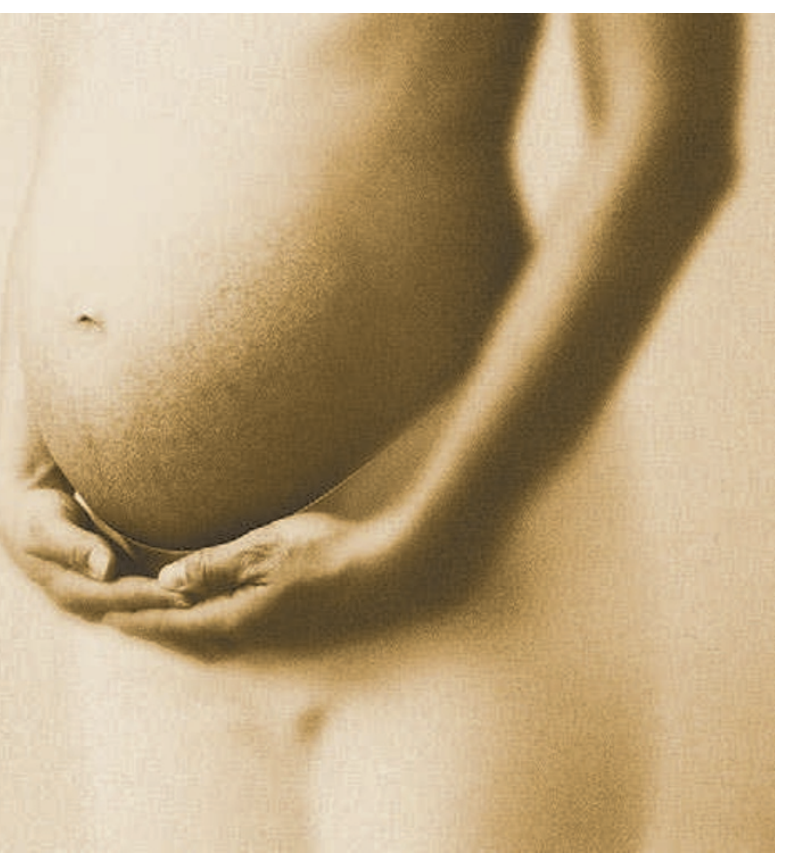

non-pathogenic bacterium Acinetobacter lwoffii F78 found in cowshed dust has been shown to protect against airway inflammation in adult mice. Now, Conrad et al. show that maternal exposure to A. lwoffii F78 protects offspring from the development of experimental asthma in mice through a mechanism that depends on maternal Toll-like receptor (TLR) signalling.

In this study, intranasal exposure of pregnant mice to A. lwoffii F78 protected their offspring from the development of ovalbumin (OVA)induced experimental asthma; this protection was characterized by decreased cell recruitment to the lungs, fewer mucus-producing goblet cells and normalization of airway reactivity compared with the progeny of mice exposed to phosphatebuffered saline. Intranasal exposure of mice to A. lwoffii F78 resulted in a transient innate immune response in the lungs and increased systemic expression of interleukin-6 (IL-6). In addition, the mRNA expression levels of TLR2, TLR3, TLR6, TLR7 and TLR9 in lung tissue were increased. By contrast, the expression of TLR6 and TLR7 mRNA was lower in the placenta of these mice, as was the expression of some cytokines.
To investigate the role of TLRs in the transfer of protection from mother to offspring, the authors generated mice that lacked expression of TLR2, TLR3, TLR4, TLR7 and TLR9 (referred to here as quintuple TLR-knockout mice). Female quintuple-knockout mice were mated with wild-type males to generate heterozygous offspring that have fully functional TLR responses. The heterozygous progeny of A. lwoffii F78-treated quintuple TLRknockout pregnant mice were not protected from experimental asthma and developed full airway inflammation and goblet cell metaplasia when sensitized and challenged with OVA. In addition, no transient innate immune response was detected in the pregnant mice following A. lwoffii F78 treatment and the downregulation of TLR expression at the placenta was absent.

So, the data indicate that maternal exposure to a farming-related microorganism can protect the offspring from the development of OVAinduced experimental asthma and identify an important role for maternal TLR expression in the transfer of this protection, possibly through the generation of a tissue-specific response in the placenta.

Olive Leavy

ORIGINAL RESEARCH PAPER Conrad, M. L. et al. Maternal TLR signaling is required for prenatal asthma protection by the nonpathogenic microbe Acinetobacter Iwoffii F78. J. Exp. Med. 7 Dec 2009 (doi:10.1084/jem.20090845) 